This is the post print version of the article, which has been published in International journal of gynecological cancer . 2017, 27 (8), 1788-1793. http://dx.doi.org/10.1097/IGC.0000000000001073 a] This document has been downloaded from TamPub uta.fi

\title{
Costs of robotic-assisted versus traditional laparoscopy in endometrial cancer
}

Riikka-Liisa K. VUORINEN, MD ${ }^{\mathrm{a}^{*}}$, Minna M. MÄENPÄÄ, MD ${ }^{\mathrm{a}^{*}}$, Kari NIEMINEN, PhD ${ }^{\mathrm{a}}$, Eija I. TOMÁS, PhDª, Tiina H. LUUKKAALA, MSc ${ }^{\mathrm{c}}$, Anssi AUVINEN, PhD ${ }^{\mathrm{d}}$, Johanna U. MÄENPÄÄ, PhD

${ }^{a}$ Department of Obstetrics and Gynecology, Tampere University Hospital, Tampere, Finland

${ }^{\mathrm{b}}$ Faculty of Medicine and Life Sciences, University of Tampere, Tampere, Finland

c Science Centre, Pirkanmaa Hospital District, Tampere, Finland and Faculty of Social Sciences, University of Tampere, Tampere, Finland

${ }^{\mathrm{d}}$ Faculty of Social Sciences, University of Tampere, Tampere, Finland

*Equal contribution

\section{Correspondence:}

Riikka-Liisa Vuorinen, Tampere University Hospital, Department of Obstetrics and Gynecology, P.O. Box 2000, FI-33521, Tampere, Finland.

Tel. +358 3 31166567, Fax +358 3 31164391, E-mail: riikka.vuorinen@pshp.fi

Funding:No funding was obtained for this study.

Word count: 2616

Clinical trial registration: ClinicalTrials.gov, www.clinicaltrials.gov, NCT01466777.

Running title: Robotic Versus Traditional Laparoscopy 


\section{Costs of robotic-assisted versus traditional laparoscopy in endometrial cancer}

\section{ABSTRACT}

Objectives The purpose of this study was to compare the costs of traditional laparoscopy and robotic-assisted laparoscopy in the treatment of endometrial cancer.

Methods/materials A total of 101 patients with endometrial cancer were randomized to the study, and operated on starting from 2010 until 2013, at the Department of Obstetrics and Gynecology of Tampere University Hospital, Tampere, Finland. Costs were calculated based on internal accounting, hospital databases and purchase prices, and compared using intention to treat analysis. Main outcome measures were item costs and total costs related to the operation, including a six month postoperative follow-up.

Results The total costs excluding late complications were $1928 €$ higher in the robotic group (median for traditional $5487 €$, vs. robot median $7415 €$, $\mathrm{p}<0.001$ ). The difference was due to higher costs for instruments, equipment as well as to more expensive operating room (OR) and post-anesthesia care unit time. Traditional laparoscopy involved higher costs for operation personnel, general costs, medication used in the operation and surgeon, although these costs were not substantial. There was no significant difference in inpatient stay, laboratory, radiology, blood products or costs related to complications.

Conclusions According to this study robotic-assisted laparoscopy is 35\% more expensive than traditional laparoscopy in the treatment of endometrial cancer. The cost difference is mainly explained by amortization of the robot and its instrumentation.

Keywords Robotic-assisted surgery, endometrial cancer, cost analysis, gynecologic oncology 


\section{Introduction}

Endometrial cancer is the most common gynecologic malignancy in the developed countries with 167,900 estimated new cases and 34,700 estimated deaths in $2012 .{ }^{1}$ Primary treatment of endometrial cancer is hysterectomy and bilateral salpingo-oophorectomy, commonly accompanied by pelvic or pelvic and para-aortic lymphadenectomy. ${ }^{2}$ Surgical methods for treating endometrial cancer include laparotomy, traditional laparoscopy and robotic-assisted laparoscopy. According to cost effectiveness analysis by Leitao et al. laparotomy was the most expensive approach compared to traditional laparoscopy and robotic-assisted laparoscopy (total costs without equipment USD 24,433, USD 20,289 and USD 20,467 respectively). ${ }^{3}$ The robot platform has been in use at Tampere University Hospital since 2009 and the robot is used by urologists, gynecologists and thoracic surgeons, with an annual rate of 345-400 operations.

The aim of this analysis was to compare the costs of conventional laparoscopy and roboticassisted laparoscopy in the treatment of endometrial cancer, to evaluate possible differences and identify factors influencing the costs within a randomized trial.

\section{Materials and Methods}

In a clinical trial 101 patients presenting with endometrial cancer were randomized into two arms, traditional laparoscopy (traditional, $\mathrm{n}=51$ ) and robotic-assisted laparoscopy (robot, $\mathrm{n}=50$ ). Inclusion criteria were a low-grade (Grade 1-2) endometrial cancer, a scheduled staging operation and a signed informed consent. Exclusion criteria included a narrow vagina or a uterus too large to be removed through the vagina and ineligibility for a deep Trendelenburg position. The details of the study population, randomization procedure and operations, have been described in detail previously. ${ }^{4}$

Costs of robotic-assisted vs. traditional laparoscopy 
The operations were performed at a tertiary referral center, the Department of Obstetrics and Gynecology of Tampere University Hospital, Tampere, Finland in 2010-2013 by gynecologic oncologists with several years of experience with laparoscopic surgery. The study protocol was approved by the Research Ethics Committee of Tampere University Hospital (identification code ETL R10081) and is registered at www.clinicaltrials.gov (NCT 01466777).

The costs were calculated retrospectively in euros. The cost variables are presented in Table 1.

The patient data were collected from the operation and onwards over the subsequent follow-up period of six months. All contacts and procedures at follow-up hospitals (imaging studies, readmissions, operative treatment) were recorded. These contacts and the costs related to them were calculated in complications and are referred in this article also as late complications. In complication costs all expenses related to the contact have been taken into account. This includes also all out-patient visits which led or did not lead to any procedures. Patients contacted clinics for various reasons like swelling, bruises and vaginal bleeding among other complaints. Most of these were normal post-operative symptoms. Because of swelling in lower extremities, many patients underwent a Doppler ultrasound imaging to exclude deep venous thrombosis, with no findings. ${ }^{4}$

Public health care in Finland uses an internal accounting and billing system within the hospitals. Different hospital units offer services based on their expertise e.g. anesthetic services, operating room (OR) services, laboratory services and consultations provided by other specialties like urologic surgery. We searched the hospital databases, in order to retrieve the actual costs of each operation.

The original expense data from 2012 was used as the basis for calculating costs for operation personnel, amortization of the laparoscopy towers and the robot console, OR-costs, medication Costs of robotic-assisted vs. traditional laparoscopy 
during the operation and general costs related to the hospital infrastructure. The 2012 expense data was chosen because it represents the mid-point of the study period.

The amortization and use of an energy instrument in traditional laparoscopy group was also included in the instrument costs; an energy instrument was used in 15 operations, based on the operating surgeon's judgment.

Costs related to the instrumentation, inpatient stay, radiology and laboratory services as well as blood products were calculated or retrieved from the database according to the actual time (exact date or at least year) of the operation. Inpatient stay, radiology, laboratory and blood product expenses were retrieved from the internal accounting system. For disposable instruments and products we used the real hospital purchase costs, according to the reported data on each operation, and we included in every operation a basic array of instruments and equipment involved in the operative set-up. Traditional and robotic operations had a different basic package based on the needs of the operative method. For reusable instruments the maintenance costs were calculated. For robot instruments the cost of amortizing (maximum 10 operations / instrument) and the maintenance costs were taken into account.

The robot at Tampere University Hospital is the Da Vinci ${ }^{\circledR}$ S surgical system (Intuitive Surgical, Inc., Sunnyvale CA). It is a leased product with a 10-year contract. The annual leasing and maintenance costs are 196,000 $€$ and 140,000 € respectively. We divided these costs with the total number of operations during the year 2012 to calculate the robot platform amortization cost per robot operation.

One patient was originally randomized into the traditional laparoscopy group, but the surgeon decided to change the operative procedure to robotic-assisted laparoscopy due to obesity of the patient. Because of this randomization violation secondary analyses were performed besides Costs of robotic-assisted vs. traditional laparoscopy 
the primary intention-to-treat analysis: A per protocol analysis with groups based on the actual operative manner (this patient included in the robot arm), and also excluding this patient.

Two patients from the traditional laparoscopy group who were not suitable for laparoscopic operation, were operated through laparotomy and their data was not analyzed in the study. ${ }^{4}$ Consequently the final number of patients in the analysis was 49 in the traditional group and 50 in the robotic-assisted group.

Distributions of cost factors were shown by medians with interquartile ranges due to the skewed distributions and outliers. Differences between traditional and robotic-assisted laparoscopic surgical costs were analyzed by non-parametric independent-samples Mann-Whitney U test. Categorical variables were tested by Pearson chi-square test or by Fisher's exact test if the expected values were too small. Statistical analyses were performed by IBM SPSS Statistics version 23 (IBM Corp., Armonk, NY). P-values less than 0.05 were considered statistically significant.

\section{Results}

Because there were no substantial differences in the results of the intention-to-treat and treatment received analyses, only the results of the intention-to-treat analysis are presented here. Results using the secondary analyses are given in the Supplemental Tables (S1-S4).

The item costs were higher in the robotic-assisted laparoscopy arm for instruments, equipment and OR, as well as post-anesthesia care unit (PACU) (Table 2). Traditional laparoscopy had higher costs for operation personnel and medication, general costs and surgeon costs, but these differences were relatively small (Table 1 for variable definitions, Table 2). There were no significant differences in costs related to inpatient stay, laboratory and radiology services or 
blood products. The median total costs for the robotic-assisted laparoscopy, including late complications were $2160 €$ higher than for traditional laparoscopy (1.4-fold, cost per operation $7982 €$ vs. $5823 €$ respectively, Fig 1$)$

There were five conversions to laparotomy in the traditional laparoscopy group and none in the robot group. ${ }^{4}$ The total costs without late complications for these patients were substantially higher than for the rest of the traditional laparoscopy patients (non-conversions Md $5352 €$ vs. conversions $7149 €, \mathrm{p}<0.001$ ) There was also a significant difference in the length of inpatient stay (Md 1 vs. 4 days, $\mathrm{p}<0.001)$, which increased the costs of the Inpatient stay (1114 € vs. $2148 €, \mathrm{p}=0.002)$. Moreover, there was a significant difference in PACU time (Md 2h22min vs. 3h33min, $\mathrm{p}<0.001$ ), which also affected the PACU costs (704 € vs. $938 € \mathrm{p}<0.001)$. The median total costs related to the laparoscopy-laparotomy converted operations are close to the median total cost of the robot arm without complications (7415 €).

Ten patients in the traditional group and 20 patients in the robot group contacted the follow-up hospitals or had complications reported. The related median costs were $766 €$ and $844 €$ per patient, respectively $(\mathrm{p}=0.530)$.

The operative time as well as the OR time were significantly shorter in the robot group, while PACU time was shorter in the traditional group (Table 3). ${ }^{4}$

Although there was no significant difference in the median length of post-operative inpatient stay (Table 3), one patient in the traditional group was not discharged until on the $7^{\text {th }}$ postoperative day. Physically the patient's recovery from the surgery did not differ from that of other patients but the patient's mental status did not allow discharge, and she was waiting for a transfer to a municipal hospital. This created an outlier in the Inpatient stay costs (3343 €). We were unable to calculate the costs of the following municipal hospital stay.

Costs of robotic-assisted vs. traditional laparoscopy 
One patient in the robot group underwent embolization while she was in the PACU due to a bleeding complication. This patient stayed in the PACU for 16h2min causing an outlier in the PACU time and PACU costs (4492 €), as well as the embolization cost in the Radiology Department (2884 €). We included the costs (embolization, laboratory, radiology, PACU) in this patient's primary operation period, not itemizing them stratified according to the complications, because it was difficult to reliably differentiate the costs of this complication from the costs of the surgery itself e.g. in the PACU costs.

One patient in the robot group needed two re-operations due to a rectovaginal-fistula. This patient also had repeated imaging studies and readmissions because of the complication. These costs created an outlier in Complication costs $(14,818 €)$.

No postoperative deaths occurred during the study period nor were there any thromboembolic events during the follow-up. ${ }^{4}$

There was no significant difference between the two arms in duration of sick-leave (Table 3). Most of the patients did not receive sick-leave as they were already retired. Consequently sickleave costs were not calculated in this analysis.

Robot instrument cost per operation was $1030 €$ (including 4 basic instruments used in the operations) and the amortization cost of the robot console per operation was $939 €$ according to the 2012 expense data (taken into account in equipment and OR costs) On this basis we calculated costs per duration of OR time related amortization cost $(5,95 € / \mathrm{min})$ and applied it individually for each operation in accordance with the operating time. Therefore the equipment and OR costs can for some patients be less than $939 €$ (range $844 €-1503 €$ ).

\section{Conclusions}

Costs of robotic-assisted vs. traditional laparoscopy 
The median actual costs of the robotic-assisted laparoscopy were 1,928 € (35\%) higher per patient than the costs related to traditional laparoscopy. Although direct international comparisons are difficult to make due to differences in national health care funding systems, our results seem to be comparable to findings in previous studies, showing robotic-assisted laparoscopy to be $17-33 \%$ more expensive. ${ }^{5,6}$ Amortization of the robot console and costs involved with robot instrumentation are the major determinants of the incremental costs related to robotic-assisted surgery. ${ }^{5-9}$ Amortization can be minimized by increasing the number of operations. However, because a set of robot instruments can only be used in 10 operations the instrument costs are practically fixed. Although we have previously shown that the operation time is shorter in robotic-assisted than traditional laparoscopic operations ${ }^{4}$, the shorter operation time was not enough to balance out the costs of amortization of the robot console and the use of robot instruments.

In Finland doctors and surgeons in the public health care receive a monthly salary instead of fee for service. This explains the lower labor cost of surgeon per operation compared to a previous US study. ${ }^{3}$ In our study the surgeon cost is related only to the duration of the operation.

The major strength of this study is the randomized design, ensuring an unbiased comparison between the treatment arms. The learning curve effect was also minimized as robotic surgery for gynecologic indications was started at our hospital already in March 2009. We have previously shown that the learning curve for robotic surgery is relatively short or 10 operations. ${ }^{10}$ Moreover our experience with laparoscopic surgery for endometrial cancer dates from 1990s. ${ }^{11}$ Both operative techniques were therefore already well-established at the time the randomized trial was initiated.

The two groups were well balanced in relation to all major patient characters. ${ }^{4}$

Costs of robotic-assisted vs. traditional laparoscopy 
The costs were calculated in a detailed fashion for each operation based on actual cost items, including even from the surgeons' gloves and threads used.

During the time of the study design, the standard surgical treatment of endometrial cancer at our institution was hysterectomy, bilateral salpingo-oophorectomy and in most cases, pelvic lymphadenectomy (PLND). These three procedures were scheduled to be performed to all of the randomized patients. PLND was not performed on two patients in both arms (total $n=4$ ) due to a disseminated disease. ${ }^{4}$

The costs of para-aortic lymphadenectomy (PALND) were not evaluated. Current guidelines encourage PALND besides PLND to be performed in patients with high-risk endometrial cancer. ${ }^{12}$ Because extending the lymphadenectomy to the para-aortic area makes traditional laparoscopy challenging to perform the cost difference might have been smaller if PALND were included in the randomized study design. ${ }^{13}$

Although the number of patients was rather limited, the outliers encountered in some variables did not substantially affect the final results.

There are some local factors that inevitably constrain the generalizability of the results. Our robot console and its PACU are located in a separate building apart from the Department of Obstetrics and Gynecology and its ORs. This increases the expenses due to additional PACU time.

Quality of life was not investigated in this study, which can be considered a limitation.

Laparoscopic approach has replaced laparotomy in the operative treatment for endometrial cancer. ${ }^{12}$ At present laparotomy should not be considered as the primary operation method anymore now that minimally invasive methods have been evolved. ${ }^{13,14}$ Also according to a Costs of robotic-assisted vs. traditional laparoscopy 
recent study comparing the costs of robotic-assisted laparoscopic hysterectomy to open hysterectomy, laparotomy was more expensive, mainly due to longer inpatient stay. ${ }^{16}$ In the field of laparoscopy, the robotic-assisted technique has introduced many advantages like diminished blood loss, wristed instruments, three dimensional stereoscopic vision, better ergonomics for surgeon, and a shorter learning curve. ${ }^{9,10,11,15,17}$ This was reflected also in the present study, where no conversions to laparotomy had to be undertaken in the robot group as opposed to five conversions in the traditional group. The total costs of the converted operations were almost as high as the costs of the robotic-assisted operations (Md $7149 €$ vs. $7415 €$ respectively).

In contrast to clinical operations performed for real patients, in which setting each robotic instrument can be used only ten times, in the preclinical training phase the same instruments can be used 30 times (data obtained during from robotic training at Tampere University Hospital, Da Vinci ${ }^{\circledR}$ S surgical system, Intuitive Surgical, Inc., Sunnyvale CA). If robotic instruments could also clinically be used 30 times it would decrease the instrument costs by $688 €$. On the other hand if the annual number of gynecological operations at our institution would be increased from 84 to 120, with three instead of two daily operation, the amortization costs would decrease by $282 €$ per operation. By such means the median total costs for robotic surgery would be $6,445 €$ and the difference between the two operation types would decrease to $17 \%$.

We were unable to assess patient outcomes in terms of quality-adjusted life-year (QALY) (because no obvious difference in complications or other patient outcomes were found), so real cost-effectiveness analysis was not possible. However, applying a cost-effectiveness threshold of 50,000 € per QALY to the observed cost difference of $2160 €$ per operation, it would mean that one QALY would need to be gained per 26 patients operated to reach the threshold.

Costs of robotic-assisted vs. traditional laparoscopy 
The robotic-assisted technique in the staging of endometrial carcinoma (hysterectomy, bilateral salpingo-oophorectomy and pelvic lymphadenectomy) increases the total treatment costs by one third compared with the traditional technique. In our setting this translates into roughly $2000 €$ per patient. For further research it would be beneficial to calculate the costs in similar form including the para-aortic lymphadenectomy. 


\section{References}

1. Torre LA, Bray F, Siegel RL, et al. Global cancer statistics, 2012. CA Cancer J Clin 2015;65:87-108.

2. Leslie KK, Thiel KW, Goodheart MJ, et al. Endometrial cancer. Obstet Gynecol Clin North Am 2012;39:255-68.

3. Leitao MM,Jr, Bartashnik A, Wagner I, et al. Cost-effectiveness analysis of robotically assisted laparoscopy for newly diagnosed uterine cancers. Obstet Gynecol 2014;123:1031-7.

4. Mäenpää MM, Nieminen K, Tomás EI, et al. Robotic-assisted vs traditional laparoscopic surgery for endometrial cancer: a randomized controlled trial. Am J Obstet Gynecol 2016;215:588.e1-588.e7

5. Barnett JC, Judd JP, Wu JM, et al. Cost comparison among robotic, laparoscopic, and open hysterectomy for endometrial cancer. Obstet Gynecol 2010;116:685-93.

6. Wright JD, Ananth CV, Tergas AI, et al. An economic analysis of robotically assisted hysterectomy. Obstet Gynecol 2014;123:1038-48.

7. Reynisson P, Persson J. Hospital costs for robot-assisted laparoscopic radical hysterectomy and pelvic lymphadenectomy. Gynecol Oncol 2013;130:95-99.

8. Lönnerfors C, Reynisson R, Persson J. A randomized trial comparing vaginal and laparoscopic hysterectomy vs robot-assisted hysterectomy. J Minim Invasive Gynecol 2015;22:78-86.

9. Schreuder HW, Verheijen RH. Robotic surgery. BJOG 2009;116:198-213.

Costs of robotic-assisted vs. traditional laparoscopy 
10. Kuoppala T, Tomas EI, Heinonen P. Clincal outcome and complications of laparoscopic surgery compared with traditional surgery in women with endometrial cancer. Arch Cynecol Obstet 2004;270:25-30.

11. Mäenpää M, Nieminen K, Tomas E, et al. Implementing robotic surgery to gynecologic oncology: The first 300 operations performed at a tertiary hospital. Acta Obstet Gynecol Scand 2015;94:482-8.

12. Colombo N, Creutzberg C, Amant F, et al,; ESMO-ESGO-ESTRO Consensus Conference on Endometrial Cancer: diagnosis, treatment and follow-up. Ann Oncol 27(1): 16-41, 2016.

13. Soliman PT, Frumovitz M, Spannuth W, et al: Lymphadenectomy during endometrial cancer staging: practice patterns among gynecologic oncologists.Gynecol Oncol 2010;119:2914)

14. Galaal K, Bryant A, Fisher AD, et al. Laparoscopy versus laparotomy for the management of early stage endometrial cancer. Cochrane Database Syst Rev 2012;9:CD006655.

15. Ramirez PT, Adams S, Boggess JF, et al. Robotic-assisted surgery in gynecologic oncology: A society of gynecologic oncology consensus statement. developed by the society of gynecologic oncology's clinical practice robotics task force. Gynecol Oncol 2012;124:180-4.

16. Herling S, Palle C, Møller A, et al. Cost-analysis of robotic-assisted laparoscopic hysterectomy versus total abdominal hysterectomy for women with endometrial cancer and atypical complex hyperplasia. Acta Obstet Gynecol Scand 2016;95:299-308.

17. Diaz-Arrastia C, Jurnalov C, Gomez G, et al. Laparoscopic hysterectomy using a computerenhanced surgical robot. Surg Endosc 2002;16:1271-3.

Costs of robotic-assisted vs. traditional laparoscopy 


\section{LEGEND FOR THE FIGURE}

Figure 1. Cost variables, comparison. (Accompanying .tif-file)

Figure 1 legend:

Median values (€)

$\mathrm{OR}=$ operating room

PACU=post-anesthesia care unit

*Nurses and an anesthesiologist

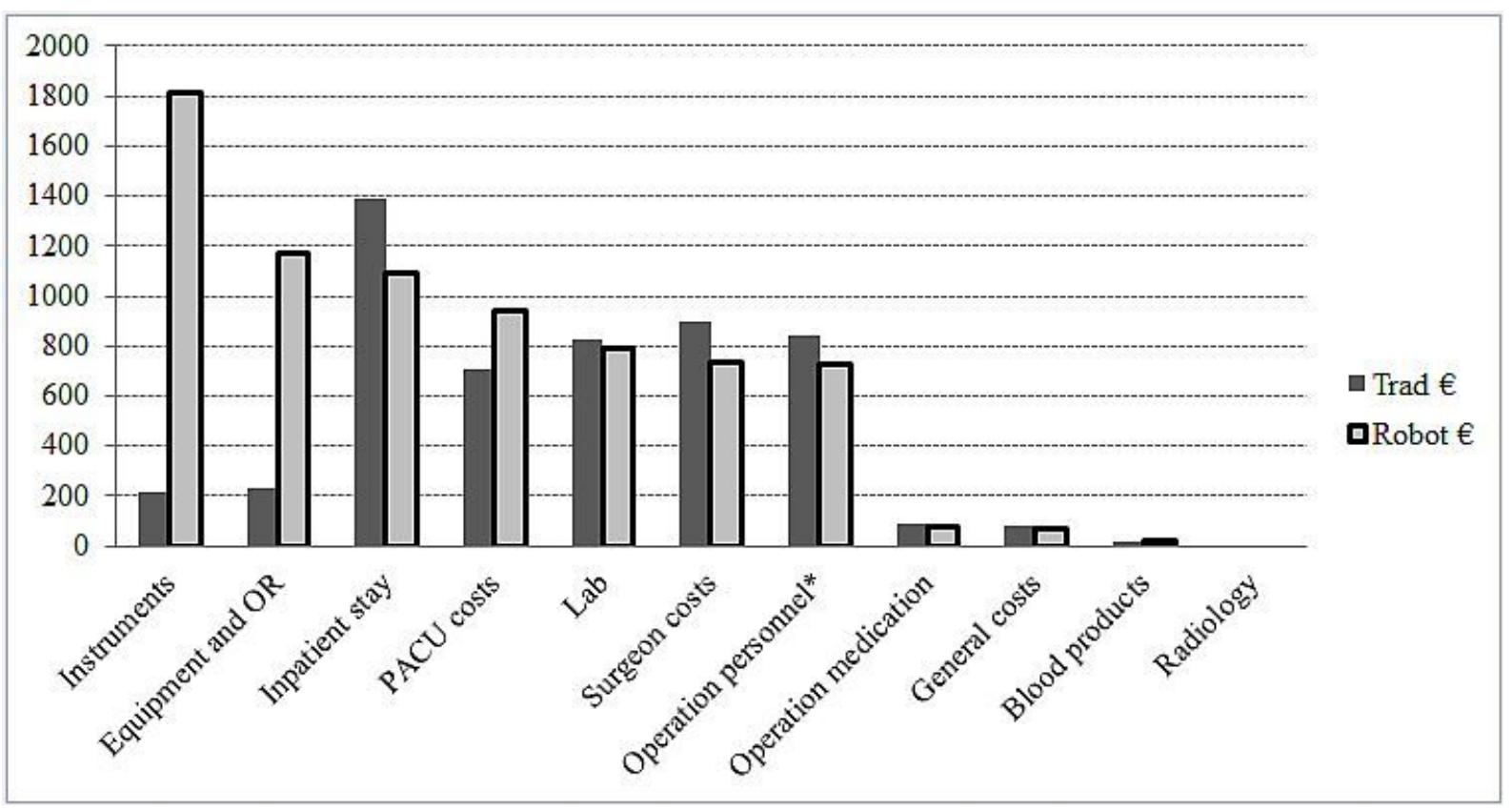




\section{Tables}

Table 1, Variable definitions.

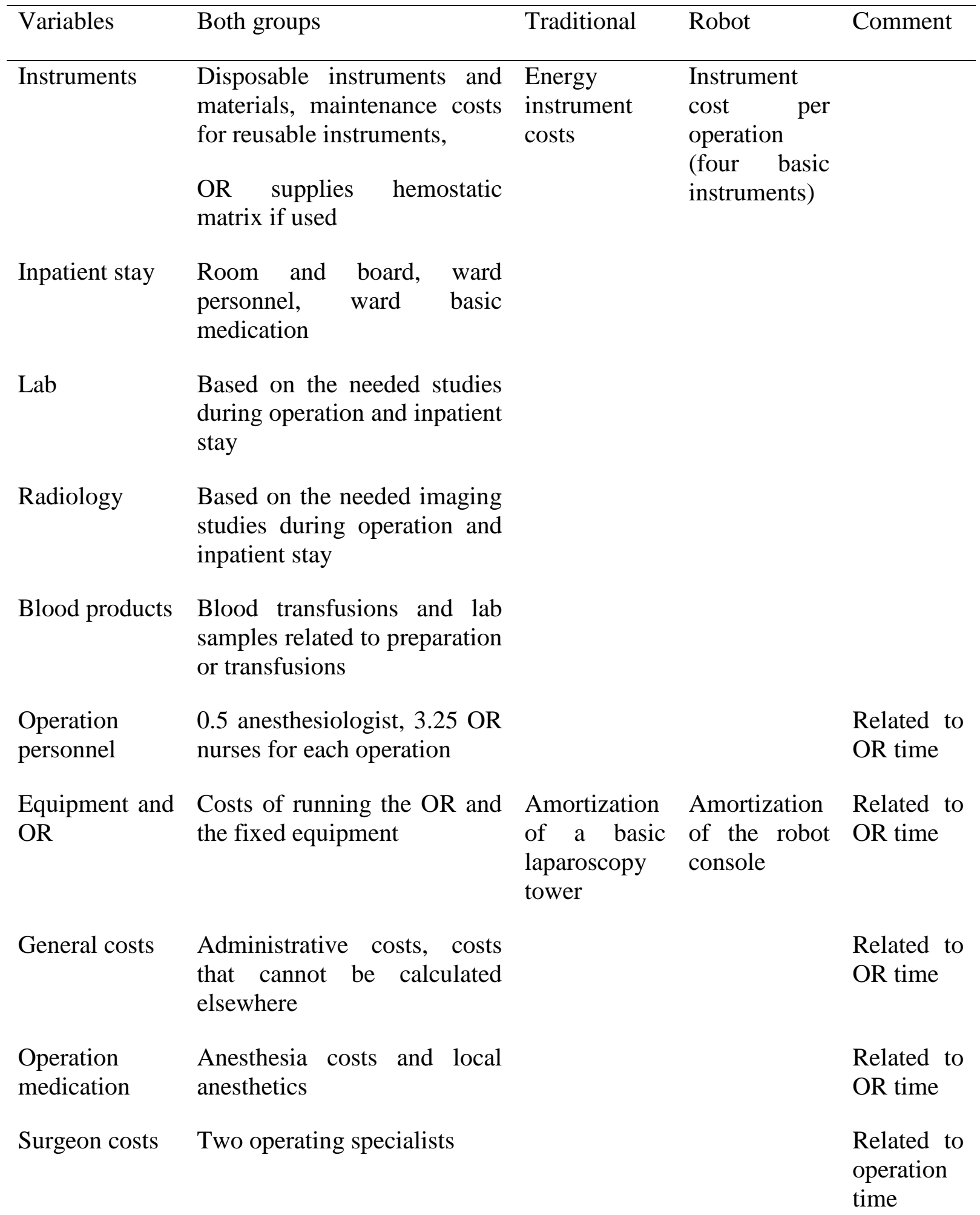

Costs of robotic-assisted vs. traditional laparoscopy 


\begin{tabular}{lll}
\hline PACU costs & $\begin{array}{l}0.3 \text { nurses per patient and } \\
\text { facilities }\end{array}$ & $\begin{array}{l}\text { Related to } \\
\text { PACU } \\
\text { time }\end{array}$
\end{tabular}

Complications Additional clinical visits, readmissions, radiology

$\mathrm{OR}=$ operating room

PACU=post-anesthesia care unit 
Table 2. Itemized median costs for traditional vs. robot-assisted laparoscopy for endometrial carcinoma, intention-to-treat analysis within the Tampere randomized trial, cost factors.

\begin{tabular}{|c|c|c|c|c|c|c|c|c|}
\hline \multirow[t]{2}{*}{ Variables } & \multicolumn{3}{|c|}{ Traditional $(n=49)$} & \multicolumn{3}{|c|}{ Robot $(n=50)$} & \multicolumn{2}{|c|}{ ifference } \\
\hline & $\mathrm{Md} €$ & $(\mathrm{IQR} €)$ & $\%$ & Md $€$ & $(\mathrm{IQR} €)$ & $\%$ & $€$ & $\mathrm{p}$ \\
\hline Instruments & 214 & $(171-421)$ & 5.9 & 1,813 & $(1,798-1,817)$ & 23.9 & $-1,599$ & $<0.001$ \\
\hline Inpatient stay & 1,387 & $(1,002-1,635)$ & 25.2 & 1,092 & $(932-1,422)$ & 15.8 & 295 & 0.130 \\
\hline Lab & 824 & $(457-918)$ & 13.3 & 791 & (526-909) & 9.3 & 33 & 0.845 \\
\hline Radiology & 0 & $(0-37)$ & 0.6 & 0 & $(0-0)$ & 1.1 & 0 & 0.321 \\
\hline Blood products & 18 & $(17-35)$ & 0.6 & 18 & $(0-40)$ & 0.6 & 0 & 0.674 \\
\hline Operation personnel* & 844 & (797-995) & 16.2 & 729 & (661-833) & 9.7 & 115 & $<0.001$ \\
\hline Equipment and OR & 232 & $(217-295)$ & 4.7 & 1,172 & $(1,064-1,340)$ & 15.7 & -940 & $<0.001$ \\
\hline
\end{tabular}




\begin{tabular}{|c|c|c|c|c|c|c|c|c|}
\hline General costs & 78 & $(73-91)$ & 1.5 & 67 & $(61-77)$ & 0.9 & 11 & $<0.001$ \\
\hline geration medication & 91 & $(86-108)$ & 1.8 & 79 & $(72-90)$ & 1.1 & 12 & $<0.001$ \\
\hline Surgeon costs & 896 & $(806-1,049)$ & 17.0 & 735 & $(643-866)$ & 9.8 & 161 & $<0.001$ \\
\hline PACU costs & 704 & (704-938) & 13.3 & 938 & (704-938) & 12.1 & -234 & $<0.001$ \\
\hline without late complications & 5,487 & $(4,766-6,184)$ & & 7,415 & $(6,937-8,057)$ & & $-1,928$ & $<0.001$ \\
\hline Complications & 766 & $(349-1,532)$ & & 844 & $(421-2883)$ & & -78 & 0.530 \\
\hline osts with co & 5,823 & $(4,912-6,243)$ & & 7,983 & $(7,236-8,400)$ & & $-2,160$ & $<0.001$ \\
\hline
\end{tabular}

Md=median value

$\mathrm{IQR}=$ interquartile range

*Nurses and an anesthesiologist 
$\mathrm{OR}=$ operating room

PACU=post-anesthesia care unit 
Table 3. Time-related items in traditional vs. robot-assisted laparoscopy for endometrial carcinoma in the Tampere randomized trial, intention-to-treat analysis.

\begin{tabular}{|c|c|c|c|c|c|}
\hline \multirow[t]{2}{*}{ Variables } & \multicolumn{2}{|c|}{ Traditional $(n=49)$} & \multicolumn{2}{|c|}{ Robot $(n=50)$} & \multirow[b]{2}{*}{$\mathrm{p}$} \\
\hline & Md & (IQR) & Md & (IQR) & \\
\hline Sick-leave, days & $27.5(n=12)$ & (24-33) & $28(n=13)$ & $(25-29.5)$ & 0.728 \\
\hline OR time & $3: 48$ & $(3: 35-4: 29)$ & $3: 17$ & $(2: 59-3: 45)$ & $<0.001$ \\
\hline Operation time & $2: 50$ & $(2: 33-3: 19)$ & $2: 19$ & $(2: 02-2: 44)$ & $<0.001$ \\
\hline PACU time & $2: 36$ & $(2: 03-3: 08)$ & 3:05 & $(2: 38-3: 31)$ & 0.001 \\
\hline Discharge, days & 2 & $(1-2)$ & 1 & $(1-2)$ & 0.215 \\
\hline
\end{tabular}

Md=median value

$\mathrm{IQR}=$ interquartile range

$\mathrm{OR}=$ operating room

PACU=post-anesthesia care unit 